\title{
Assigning Types to Processes
}

\section{(Extended Abstract)}

\author{
Nobuko Yoshida \\ ny11@mcs.le.ac.uk \\ MCS, University of Leicester, UK
}

\author{
Matthew Hennessy \\ matthewh@cogs.susx.ac.uk \\ COGS, University of Sussex, UK
}

\begin{abstract}
In wide area distributed systems it is now common for higher-order code to be transferred from one domain to another; the receiving host may initialise parameters and then execute the code in its local environment. In this paper we propose a fine-grained typing system for a higher-order $\pi$ calculus which can be used to control the effect of such migrating code on local environments. Processes may be assigned different types depending on their intended use. This is in contrast to most of the previous work on typing processes where all processes are typed by a unique constant type, indicating essentially that they are well-typed relative to a particular environment.

Our process type takes a form of an interface limiting the resources to which it has access, and the types at which they may be used. Allowing resource names to appear both in process types and process terms, as interaction ports, complicates the typing system considerably. For the development of a coherent typing system, we use a kinding technique, similar to that used by the subtyping of the system $F$, and order-theoretic properties of our subtyping relation.

Various examples of this paper illustrate the use of our fine-grained typing system for distributed systems. As a specific application we define a new typed behavioural equivalence for the higher-order $\pi$-calculus. The expressiveness of our types enables us to state and prove interesting identities between typed processes.
\end{abstract}

\section{Introduction}

Background In the distributed computing environments nowadays, it is common for higher-order code to be transferred from one domain to another [10, 23, 25]. This is recognised as dangerous and various schemes have been put forward to ensure the integrity of systems in the presence of such operations. In this paper we propose a new subtyping system which can be used to control the effect of migrating code on local environments. Our investigation is in terms of a higher-order $\pi$-calculus in which values, including process terms, can be exchanged along communication channels $[3,29,37]$. We believe that our typing system can be readily adapted to related location based distributed calculi such as $[5,8,18,12,28,34]$.

Higher-Order Processes The language we consider, $\lambda \pi_{v}$, is essentially a call-by-value $\lambda$-calculus [9] augmented with the $\pi$-calculus primitives [22]. For example,

$$
c ?(x: \tau) f x
$$

is a process which inputs a value of type $\tau$ on channel $c$ and applies to it the function $f$. This process will be welltyped only in an environment in which the channel $c$ has the capability to input values of type $\tau$, written $c:(\tau)^{\mathrm{I}}$, and $f$ denotes a function of type ( $\tau \rightarrow$ proc); here, as in [26, 37], we use proc to denote the type of processes.

As usual we allow as values arbitrary abstractions, but much of the descriptive power of $\lambda \pi_{\mathrm{v}}$ comes from the ability to form values by abstracting over processes. For example (unit $\rightarrow$ proc) is the type of thunked processes; we use this type so frequently that we will abbreviate it to $\langle$ proc $\rangle$. Values of this type, of the form $\lambda$ ( $x$ : unit).P, will also be abbreviated to $\langle P\rangle$. Such values can be exchanged between processes and subsequently executed, by applying the function $\lambda y . y()$; again for the sake of clarity we use run to denote this function. So in (1) above, if $\tau$ is the thunked type $\langle$ proc $\rangle$ and $f$ is the function run, the process may input a thunked process $\langle P\rangle$ on channel $c$ and execute it.

In papers such as $[3,21,26,27,33,37]$ typing systems have been suggested which ensure that programs written in $\lambda \pi_{\mathrm{v}}$-related languages are well-behaved. The main judgements normally take the form

$$
\Gamma \vdash P: \text { proc }
$$

indicating that the term $P$ is a well-typed process relative to the typing environment $\Gamma$. Here $\Gamma$ is a mapping from channel names or variables to input/output capabilities or value types; $\Gamma(c)$ determines the type of values which channel $c$ may transmit/receive. Thus " $c$ ? $(x: \tau) f x$ " in (1), will be well-typed in any environment $\Gamma$ which allows $c$ the input capability $\langle$ proc $\rangle$, assuming of course that $f$ is also a well-typed expression of type $\langle$ proc $\rangle \rightarrow$ proc, such as $\Gamma \supset\left\{c:\langle\text { proc }\rangle^{\mathrm{I}}, f:\langle\right.$ proc $\rangle \rightarrow$ proc $\}$.

However such typing offers limited control to programs over the code which they download for execution. To emphasise this point let us consider an example. First we de- 
fine the abstraction Fw (called a forwarder in [16])

$$
\lambda x \lambda y(* x ?(z: \text { int }) y !\langle z\rangle)
$$

which repeatedly inputs some value of a type int on channel $x$ and outputs it immediately on $y$. If the channels $a, b$ are assigned suitable types then both the values $\left\langle\mathrm{Fw}_{\mathrm{w}}(a b)\right\rangle$ and $\langle\mathrm{Fw}(b a)\rangle$ have type $\langle$ proc $\rangle$ and thus may be sent along channel $c$ to a process such as

$$
c ?(x: \tau) \text { run } x
$$

But accepting these processes for execution confers on the incoming code different access rights. In the first case the incoming code is allowed to read from channel $a$ and write to channel $b$ while in the second case these rights are reversed. Typing systems in which code can only be assigned the undifferentiated type proc do not provide any mechanism for limiting the effect of incoming code.

Typing processes In this paper we extend the typing systems of $[37,26,12]$ by allowing processes to have types which bound the resources which they may use. The basic idea is straightforward. For processes in $\lambda \pi_{\mathrm{v}}$ we will allow judgements of the form

$$
\Gamma \vdash P:[\Delta]
$$

where $\Delta$ is a finite environment, mapping channel names to capabilities. Intuitively this means that relative to $\Gamma$ the term $P$ denotes a well-defined process which uses at most the resources in the domain of $\Delta$; moreover their use is in accordance with the capabilities given in $\Delta$. Thus the type $[\Delta]$ may be viewed as a process interface.

For example let $\Delta_{a b}, \Delta_{b a}$ denote the environments

$$
\left\{a:(\text { int })^{\mathrm{I}}, b:(\text { int })^{\mathrm{D}}\right\}, \quad\left\{b:(\text { int })^{\mathrm{I}}, a:(\text { int })^{\mathrm{D}}\right\}
$$

respectively where $(\text { int })^{\mathrm{I}}$ and (int $)^{\mathrm{O}}$ represent the input and output capabilities of a type int. Then, for a suitable $\Gamma$ we will be able to derive the judgements

$$
\Gamma \vdash \operatorname{Fw}(a b):\left[\Delta_{a b}\right] \quad \text { and } \Gamma \vdash \operatorname{Fw}(b a):\left[\Delta_{b a}\right]
$$

These more discriminating types for processes allow processes to be, in turn, more discriminating in the type of values which they will accept. Thus

$$
c ?\left(x:\left\langle\Delta_{a b}\right\rangle\right) \text { run } x
$$

indicates that it is only willing to accept processes for execution if they at most read from resource $a$ and write to $b$. Let us denote $c !\langle P\rangle$ for an output process which sends a thunked process $\langle P\rangle$ to a channel $c$. Thus, for example, a process

$$
c !\langle\mathrm{Fw}(a b)\rangle \mid c ?\left(x:\left\langle\Delta_{a b}\right\rangle\right) \operatorname{run} x
$$

is well-typed while

$$
c !\langle\mathrm{Fw}(b a)\rangle \mid c ?\left(x:\left\langle\Delta_{a b}\right\rangle\right) \text { run } x
$$

is not; the (thunked) process $\langle\mathrm{Fw}(b a)\rangle$ is not acceptable along $c$ as it does not conform to the interface decreed by the host, $\Delta_{a b}$.
This ability to constrain the effect of imported code means that host processes can maintain the consistency of local resources. For example consider the following example: nesting process types give even further control over code behaviour.

*req $?\left(y:\left\langle\Delta_{c}\right\rangle\right)\left(\right.$ run $y \mid c ?\left(x:\left\langle\Delta_{a}\right\rangle\right)(\operatorname{run} x \mid a ?(z$ : int $\left.) P)\right)$

where $\left\langle\Delta_{a}\right\rangle$ denotes the type $\left.\langle a \text { : (int })^{0}\right\rangle$ and $\left\langle\Delta_{c}\right\rangle$ denotes $\left\langle c:\left\langle\Delta_{a}\right\rangle^{0}\right\rangle$. The annotated types ensure that (1) the code downloaded on the request channel req can only access the resource $c$, (2) $c$ can only be used to transmit code which can at most access resource $a$, and (3) all communications to $a$ will be serviced by $a$ ? $(z$ : int $) P$.

Channel abstractions In $\lambda \pi_{\mathrm{v}}$ processes are also allowed to download abstracted code; code in which resource parameters may be instantiated by the host process before the code is executed. A simple example is the abstraction Fw used above. Consider the server

$$
* s ?(z) z !\langle\mathrm{Fw}\rangle
$$

which continually supplies the abstraction Fw to requesting clients. A specific client, such as $R$ defined by

$$
s !\langle c\rangle c ?(y)(y a b)
$$

can download Fw and instantiate it with particular channels, such as $a, b$. Thus in the presence of the server $R$ will evolve to a process which should have a type of the form

$$
\left[a:(\text { int })^{\mathrm{I}}, b:(\text { int })^{\mathrm{D}}, \ldots\right]
$$

Other processes which instantiate $\mathrm{F}_{w}$ differently will evolve to processes with different types. For example $S$ defined by

$$
s !\langle c\rangle c ?(y)(y b a)
$$

will evolve to a process with a type

$$
\left[b:(\text { int })^{\mathrm{I}}, a:(\text { int })^{\mathrm{D}}, \ldots\right]
$$

However it is difficult to see how to give a type to the abstraction Fw which ensures that $R$ and $S$ are assigned such types. Within our current system of types it would be natural to assign to $F_{w}$ a functional type of the form

$$
(\mathrm{int})^{\mathrm{I}} \rightarrow(\mathrm{int})^{\mathrm{D}} \rightarrow \pi
$$

for some process type $\pi$. If $\pi$ is the undifferentiated type proc then both $R$ and $S$ would inherit this uninformative type. Otherwise $\pi$ must assign some definite capabilities to $a$ and $b$ and assuming that typing is preserved under Subject Reduction these capabilities would be inherited by $R$ and $S$. That is, they would have the same capabilities on the two resources $a$ and $b$, contrary to our requirements.

Our solution is to introduce a new form of dependent functional type

$$
(x: \sigma) \rightarrow \rho
$$

Here $\sigma$ is a channel type and we allow the type $\rho$ to contain occurrences of the channel variable $x$. (These occurrences 
of $x$ in $\rho$ are bound occurrences in $(x: \sigma) \rightarrow \rho$.) Thus the abstraction $F w$ will be assigned the type

$$
\left(x:(\text { int })^{\mathrm{I}}\right) \rightarrow\left(y:(\text { int })^{\mathrm{D}}\right) \rightarrow\left[x:(\text { int })^{\mathrm{I}}, y:(\text { int })^{\mathrm{D}}\right]
$$

where the result type of the process depends on the type of the abstracted variables.

Results In this paper we formulate a fine-grained subtyping system for the higher-order $\pi$-calculus following the basic ideas mentioned above. This results in a simple, but nontrivial, extension of the IO-subtyping for $\pi$-calculus [26] to the higher-order setting. The main technical results can be summarised as follows.

1. First, we design a novel notion of type for the higherorder $\pi$-calculus, in which process have types corresponding to interfaces. Because type variables appear both in program terms and in types (in particular the types of processes), the formal definition of what constitutes a valid term and a valid type are interdependent and both in turn require a careful definition of even a valid typing environment. An analogous, albeit somewhat simpler, situation arises in subtyping for the polymorphic $\lambda$-calculus [6]. We clarify technical similarities and differences between the two typing systems. Although some techniques developed for the $\lambda$-calculus are useful in the current setting, novel concepts are required for process types.

2. Second, we propose a typing system in which many higher-order $\pi$-calculus processes can be assigned non-trivial interface types. We prove its soundness (Subject Reduction Theorem) and also establish a Type Safety Theorem, which ensures that no well-typed process can input higher-order code which does not conform the local interface of that process.

3. Finally we define a new typed behavioural equivalence for the higher-order $\pi$-calculus. Our fine-grained types enable us to establish general, and useful, process identities. Their application is illustrated on an example distributed system.

Outline of the Paper Section 2 introduces the types, syntax and a reduction semantics of $\lambda \pi_{\mathrm{v}}$. Section 3 proposes the new typing system of $\lambda \pi_{\mathrm{v}}$ and demonstrates its expressiveness by typing the example in Section 2. In Section 4, we prove Subject Reduction and Type Safety Theorems. Section 5 briefly studies the typed behavioural equalities. Finally Section 6 concludes the paper with further issues and related work. Due to space limitations, the proofs and many examples, including a further application to a distributed higher-order $\pi$-calculus, are left to the full version [38].

\section{A Higher-order Process Language}

Types The collection of types is a straightforward extension of that from [37]; The formal definition is given in Figure 1; this assumes a set of base types such as unit and nat, an infinite set of channel or names $\mathbf{N}$, ranged over by $a, b, \ldots$, and an infinite set of variables $\mathbf{V}$, ranged over by $x, y, \ldots$ For the sake of clarity we will sometimes use $X, Y, \ldots$ as variables, whenever we intend them to be substituted specifically by higher order values rather than channels.

Channel types are as in [37], in turn an elaboration of the IO-types of $[12,26]$; they take the form $\left\langle S_{\mathrm{I}}, S_{0}\right\rangle$, a pair consisting of an input sort $S_{\mathrm{I}}$ and an output sort $S_{0}$; these input/output sorts are in turn either a general value type or $T$, denoting the highest capability, or $\perp$, denoting the lowest; as explained in [37] the representation of IO-types as a tuple $[13,12]$ makes the integration with the arrow types of the $\lambda$-calculus more natural. Moreover the IO-types of [26] can also be represented as a special case of our IO-types, using the abbreviations given in Figure 1 .

There are three kinds of value types: base types, channel types as already explained or HO-value types, ranged over by $\sigma_{H}$. These can be formed using either of the functional type constructors, $\sigma_{H} \rightarrow \rho$ or $(x: \sigma) \rightarrow \rho$, where $\rho$ in turn is either a HO-value type or a process type. Process types can either be the constant proc (also denoted $\circ$ in [26]), or a type environment $[\Delta]$ where $\Delta$ is a mapping from $\mathbf{N} \cup \mathbf{V}$ to channel types; the formation rules for environments are also given in Figure 1.

\section{Example 2.1 (Types)}

(1) The nil process, with no capabilities, has the type [ ].

(2) A process which can output nat at $a$ and input bool at $b$ has the type $\left[a:(\text { nat })^{\mathrm{D}}, b:(\mathrm{bool})^{\mathrm{I}}\right]$.

(3) A HO process which can output a thunked value of type (2) at $c$ has the type $\left[c:\left\langle a:(\text { nat })^{0}, b:(\text { bool })^{\mathrm{I}}\right\rangle^{0}\right]$.

(4) A higher order identity function over thunked values of type (2) has the type $\left\langle a:(\text { nat })^{\mathrm{D}}, b:(\text { bool })^{\mathrm{T}}\right\rangle \rightarrow\left\langle a:(\text { nat })^{\mathrm{D}}, b:(\text { bool })^{\mathrm{T}}\right\rangle$.

(5) A dependent function which is applied to some name $a$ and constructs a process of type $\left[b:(\text { nat })^{\mathrm{I}}, a:(\text { nat })^{\mathrm{D}}\right]$ has the type $\left(x:(\text { nat })^{0}\right) \rightarrow\left[b:(\text { nat })^{\mathrm{I}}, x:(\text { nat })^{\mathrm{D}}\right]$.

Syntax The syntax for terms in the language $\lambda \pi_{\mathrm{v}}$ is given in Figure 2. It is essentially the same as that used in [37] except that we use the more expressive types, from Figure 1 . We use the standard notational conventions, for example ignoring trailing occurrences of the empty process $\mathbf{0}$ and omitting type annotations unless they are relevant. We also define the notions of free names $\mathrm{fn}(P)$ and free variables $\mathrm{fv}(P)$ of terms which may appear in the annotated types. The formal definitions are available in the Appendix of [38], but as an example fv $\left(u ?\left(x_{1}: \tau_{1}, \ldots, x_{n}\right.\right.$ : $\left.\left.\tau_{n}\right) P\right)=\mathrm{fv}(u) \cup \mathrm{fv}\left(\tau_{1}\right) \cup \ldots \cup \mathrm{fv}\left(\tau_{n}\right) \cup \mathrm{fv}(P)-\left\{x_{1}, \ldots, x_{n}\right\}$.

Reduction Semantics The term $P$ is called a program if it contains no free variables, i.e. $\operatorname{fv}(P)=\emptyset$. The reduction semantics is given in terms of a binary relation

$$
P \longrightarrow Q
$$

between programs and follows the standard approach from $[22,26,29]$; the formal definition is given in Figure 3 and 
(Type) $\alpha, \beta, \gamma, \ldots$

Term: $\quad \rho \quad::=\pi \mid \sigma_{H}$

Base: $\quad \sigma_{B}::=$ unit $\mid$ nat $\mid \cdots$

Process: $\pi \quad::=[\Delta] \mid$ proc

HO Value: $\quad \sigma_{H}::=\sigma_{B}\left|\sigma_{H} \rightarrow \rho\right|(x: \sigma) \rightarrow \rho$

Channel: $\quad \sigma \quad::=\left\langle S_{\mathrm{I}}, S_{0}\right\rangle$

Value: $\quad \tau \quad::=\sigma_{H} \mid \sigma$

Sort: $\quad S \quad::=\left(\tau_{1}, \ldots, \tau_{n}\right)|\top| \perp$
(Environment)

Channel:

$\Delta::=\emptyset \mid \Delta, u: \sigma$

General:

$\Gamma::=\emptyset|\Gamma, x: \tau| \Gamma, a: \sigma$
(Abbreviation)

input only: $\quad S^{\mathrm{I}} \stackrel{\text { def }}{=}\langle S, \perp\rangle$ output only: $\quad S^{\mathrm{D}} \stackrel{\text { def }}{=}\langle\top, S\rangle$

input/output: $\quad S^{\text {IO }} \stackrel{\text { def }}{=}\langle S, S\rangle$

thunk type: $\langle\Delta\rangle \stackrel{\text { def }}{=}$ unit $\rightarrow[\Delta]$

Figure 1. Types

(Term)

$\begin{aligned} P, Q, \ldots::= & V \\ & \mathbf{0} \\ & P \mid P \\ & u !\left\langle V_{1}, \ldots, V_{n}\right\rangle P \\ & u ?\left(x_{1}: \tau_{1}, \ldots, x_{n}: \tau_{n}\right) P \\ & * P \\ & (\vee a: \sigma) P \\ & P P\end{aligned}$

(Identifier)

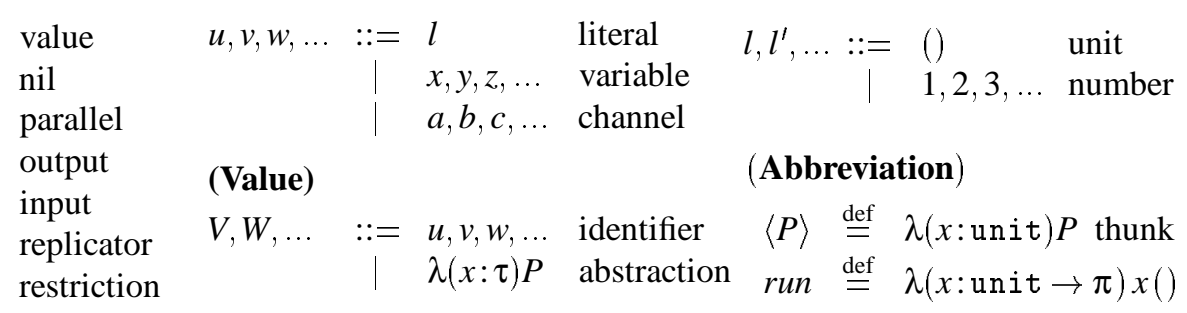

application

\section{(Literal)}

Figure 2. Syntax

\section{(Reduction)}

( $\beta)$

$\left(\operatorname{app}_{r}\right) \frac{Q \longrightarrow Q^{\prime}}{P Q \longrightarrow P Q^{\prime}} \quad\left(\operatorname{app}_{l}\right) \frac{P \longrightarrow P^{\prime}}{P V \longrightarrow P^{\prime} V}$

(com) $a ?\left(x_{1}: \tau_{1}, \ldots, x_{n}: \tau_{n}\right) P \mid a !\left\langle V_{1}, \ldots, V_{n}\right\rangle Q$

$$
\begin{aligned}
& \quad \longrightarrow P\left\{V_{1}, \ldots, V_{n} / x_{1}, \ldots, x_{n}\right\} \mid Q \\
& \text { (par) } \frac{P \longrightarrow P^{\prime}}{P\left|Q \longrightarrow P^{\prime}\right| Q} \quad \text { (res) } \frac{P \longrightarrow P^{\prime}}{(\text { v } a: \sigma) P \longrightarrow(\text { v } a: \sigma) P^{\prime}} \\
& \text { (str) } \frac{P \equiv P^{\prime} \longrightarrow Q^{\prime} \equiv Q}{P \longrightarrow Q}
\end{aligned}
$$

\section{(Structure Equivalence)}

- $P \equiv Q$ if $P \equiv_{\alpha} Q$.

- $P|Q \equiv Q| P \quad(P \mid Q)|R \equiv P|(Q \mid R) \quad P \mid \mathbf{0} \equiv P$ $* P \equiv P \mid * P$

- $(v a: \sigma) \mathbf{0} \equiv \mathbf{0}$

$(v a: \sigma) P \mid Q \equiv(v a: \sigma)(P \mid Q)$ if $a \notin \mathrm{fn}(Q)$

$(v a: \sigma)\left(v b: \sigma^{\prime}\right) P \equiv\left(\vee b: \sigma^{\prime}\right)(v a: \sigma) P$ if $a \notin \mathrm{fn}\left(\sigma^{\prime}\right)$ and $b \notin \mathrm{fn}(\sigma)$

Figure 3. Reduction

should be understandable to those familiar with either the $\pi$-calculus or the $\lambda$-calculus. It uses the standard structural equivalence $\equiv$ of the $\pi$-calculus; the axioms for $\equiv$ is also given in Figure 3. We also use $\rightarrow$ to denote the multi-step reduction. The main rules are $\beta$-reduction, $(\beta)$, and communication (com). Both these require a definition of substitution of values for variable $P\{V / x\}$, which we have yet to define. Complications arise when the value to be substituted $V$ is a channel name and is best explained with an example:

$$
\begin{aligned}
\lambda\left(x:(\text { int })^{\mathrm{IO}}\right) \cdot \lambda\left(Y:\left\langle x:(\text { int })^{\mathrm{I}}, a:(\text { int })^{\mathrm{D}}\right\rangle\right) \\
(\text { run } Y|x !\langle 1\rangle| a ?(z) r !\langle z\rangle)
\end{aligned}
$$

This function first takes some channel, say $b$, then takes a thunked process with a $b$-capability and an $a$-capability, sets it running and interacts with it via $a$ and $b$. Suppose that it is applied to the specific channel $b$. Intuitively this means substituting the value $b$ for free occurrences of the bound variable $x$ in the body of the function. If the substitution ignores types in the body of the function, we get

$$
\lambda\left(Y:\left\langle x:(\text { int })^{\mathrm{I}}, a:(\text { int })^{0}\right\rangle\right)(\text { run } Y|b !\langle 1\rangle| a ?(z) r !\langle z\rangle)
$$

which is not even a program; it contains an occurrence of the free variable $x$. The proper definition of reduction requires that $b$ is also substituted into the types occurring in the body of the function, to give the program

$$
\lambda\left(Y:\left\langle b:(\text { int })^{\mathrm{I}}, a:(\text { int })^{0}\right\rangle\right)(\text { run } Y|b !\langle 1\rangle| a ?(z) r !\langle z\rangle)
$$

This also makes sense as this now constrains the function to be only applied to processes which have a $b$-capability.

The formal definition of value substitution into terms, $P\{V / x\}$, is defined inductively on the structure of terms. 


$$
\begin{array}{rll}
\top\{v / x\}=\top & & \perp\{v / x\}=\perp \\
\sigma_{B}\{v / x\}=\sigma_{B} & & \operatorname{proc}\{v / x\}=\operatorname{proc} \\
\left\langle S_{\mathrm{I}}, S_{0}\right\rangle\{v / x\} & =\left\langle S_{\mathrm{I}}\{v / x\}, S_{0}\{v / x\}\right\rangle, \\
\left(\tau_{1}, \ldots, \tau_{n}\right)\{v / x\} & =\left(\tau_{1}\{v / x\}, \ldots, \tau_{n}\{v / x\}\right) \\
\left(\sigma_{H} \rightarrow \rho\right)\{v / x\} & =\sigma_{H}\{v / x\} \rightarrow \rho\{v / x\} \\
((y: \sigma) \rightarrow \rho)\{v / x\} & =(y: \sigma\{v / x\}) \rightarrow \rho\{v / x\}(x \neq y) \\
{[\Delta]\{v / x\}} & =\sqcup[w\{v / x\}: \sigma\{v / x\}](w: \sigma \in \Delta)
\end{array}
$$

Figure 4. Name Substitution into Types

The following is one instance for an input process:

$$
u ?(x: \tau) P\{V / x\} \stackrel{\text { def }}{=} u\{V / x\} ?(x: \tau\{V / x\}) P\{V / x\}
$$

However this instance uses the substitution of values into types, $\tau\{V / x\}$. If $V$ is anything other than a channel name or variable this is the identity. So we need only define the substitution $\tau\{v / x\}$, where $v$ is a channel or channel variable; this is defined in Figure 4.

This definition of substitution into types is for the most part straightforward, with one exception which can be explained using the example function (3) above. If this is applied to the name $a$ we would expect to get the result

$$
\lambda\left(Y:\left\langle a:(\text { int })^{\mathrm{IO}}\right\rangle\right)(\operatorname{run} Y|a !\langle 1\rangle| a ?(z) r !\langle z\rangle)
$$

since $a$ is allowed to have an input/output capability (int) ${ }^{\text {IO }}$ in the body. In other words the substitution of the name $a$ for $x$ in the type $\left.\left\langle x:(\text { int })^{\mathrm{I}}, a: \text { (int }\right)^{\mathrm{D}}\right\rangle$ should be $\left\langle a:(\text { int })^{\mathrm{IO}}\right\rangle$. This is reflected in the final clause in Figure 4:

$$
[\Delta]\{v / x\}=\sqcup[w\{v / x\}: \sigma\{v / x\}] \text { with } w: \sigma \in \Delta
$$

Here $\sqcup$ is an operator on types which intuitively acts like a (partial) least upper bound with respect to a yet to be defined subtyping order on types. The following are simple examples of $\sqcup$ on process types, which may be sufficient to read this paper; roughly speaking, $\sqcup$ calculates the union of the accessibility rights of two processes. (The formal definition is available in the Appendix in [38].)

$$
\begin{aligned}
& {\left[a:(\text { int })^{\mathrm{I}}\right] \sqcup\left[b:(\text { int })^{\mathrm{O}}\right]=\left[a:(\text { int })^{\mathrm{I}}, b:(\text { int })^{\mathrm{O}}\right] \text { and }} \\
& {\left[a:(\text { int })^{\mathrm{I}}\right] \sqcup\left[a:(\text { int })^{\mathrm{O}}\right]=\left[a:(\text { int })^{\mathrm{IO}}\right]}
\end{aligned}
$$

Now we can analyse the substitution on types in the above example by the following equations.

$$
\begin{aligned}
& {\left[x:(\text { int })^{\mathrm{I}}, a:(\text { int })^{\mathrm{O}}\right]\{b / x\}} \\
& =\left[x\{b / x\}:(\text { int })^{\mathrm{I}}\right] \sqcup\left[a:(\text { int })^{\mathrm{O}}\right]=\left[b:(\text { int })^{\mathrm{I}}, a:(\text { int })^{\mathrm{O}}\right] \\
& {\left[x:(\text { int })^{\mathrm{I}}, a:(\text { int })^{\mathrm{O}}\right]\{a / x\}} \\
& =\left[x\{a / x\}:(\text { int })^{\mathrm{I}}\right] \sqcup\left[a:(\text { int })^{\mathrm{O}}\right]=\left[a:(\text { int })^{\mathrm{IO}}\right]
\end{aligned}
$$

The properties of $\sqcup$ will be discussed in the next section, when we consider well-typed programs. Note that in general $\sqcup$ is a partial operator and therefore apriori substitution is not always defined. However we will see that in properly typed environments it is always well-defined.

Example 2.2 (Interface server and mobile client code) A (specific) compute service is a process which given

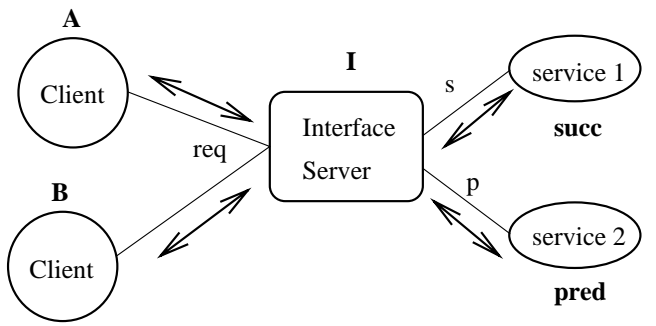

Figure 5. Interface Server and Distributed Services

some data and a return address, applies some specified operation to the data and returns the result to the address. For some given name $a$, let $\operatorname{Succ}(a)$ represent the process $* a ?(y, z) z !\langle\operatorname{succ}(y)\rangle$, which we write as

$$
\operatorname{Succ}(a) \Longleftarrow * a ?(y, z) z !\langle\operatorname{succ}(y)\rangle
$$

This represents a service (for succ) situated at $a$. A similar one for the predecessor function, pred, is defined as:

$$
\operatorname{Pred}(a) \Longleftarrow * a ?(y, z) z !\langle\operatorname{pred}(y)\rangle
$$

A client may wish a number of operations to be performed on given data, in a particular sequence, with some data for later operations depending on results produced by earlier operations. This situation is given diagrammatically in Figure 5. Here there is an Interface (I) between clients and the collection of services or operations on offer. Now a higherorder script is sent to the interface. This script is executed locally by the interface, which interacts as necessary with the various services. This protocol puts the computational onus on the server and avoids repeated interactions between clients and services. The server at the interface $I$ may then be defined by:

$$
\operatorname{Serv}_{I}(\text { req, } s, p) \Longleftarrow \quad * \text { req? }(X) X s p
$$

It takes in a script $X$, a process parameterised on service ports, and applies it to the actual port names of the two local services, in this case $s$ and $p$. Note that these actual names are not known to clients, thereby, in this case, affording some security protection to the server from clients; all interaction between clients and the server is through the interface req.

We give two examples of clients requesting services. Client (A) wants to increment a number $k$ twice, whereas the Client (B) wants to evaluate the successor and the predecessor of two different numbers $n$ and $m$ in parallel.

$$
\begin{aligned}
& \mathrm{C}_{A}(\mathrm{req}) \Longleftarrow \\
& \quad \text { req! }\left\langle\lambda(s, p)\left((v c) s !\langle k, c\rangle c ?(z) s !\langle z, c\rangle \mathrm{Fw}\left(c r_{A}\right)\right)\right\rangle \\
& \mathbf{C}_{B}(\text { req }) \Longleftarrow \\
& \text { req! }\left\langle\lambda(s, p)\left(v c c^{\prime}\right)\left(s !\langle n, c\rangle \mathrm{Fw}\left(c r_{1 B}\right) \mid p !\left\langle m, c^{\prime}\right\rangle \mathrm{Fw}\left(c^{\prime} r_{2 B}\right)\right)\right\rangle
\end{aligned}
$$

The forwarders in their bodies are used to relay the final results to each client on their result channels, $r_{A}$ for Client (A), and $r_{1 B}, r_{2 B}$ for Client (B) respectively. 
Putting the clients and server together we have the following parallel composition (Figure 5):

$$
\mathbf{C}_{A}(\mathrm{req})\left|\mathbf{C}_{B}(\mathrm{req})\right| \operatorname{Serv}_{I}(\mathrm{req}, s, p)|\operatorname{Succ}(s)| \operatorname{Pred}(p)
$$

After a certain amount of reductions, $k+2$ is returned to Client (A) on $r_{A}$, and $n+1$ and $m-1$ are returned to Client (B) on $r_{1 B}$ and $r_{2 B}$, respectively.

\section{The Fine-Grained Typing System}

\subsection{Well-formed Types and Environments}

In Figure 6 we present a formal system with three forms of judgements, all interrelated:

$$
\begin{array}{lll}
\Gamma & \vdash \text { Env } & \Gamma \text { is a well-formed environment } \\
\Gamma \quad \vdash \alpha: \operatorname{tp} & \alpha \text { is a well-formed type in } \Gamma \\
\Gamma \quad \vdash \alpha \leq \alpha^{\prime} & \alpha \text { is less than } \alpha^{\prime} \text { in } \Gamma
\end{array}
$$

For convenience we use $\Gamma \vdash \mathrm{J}$ as a shorthand for any of the three allowed forms of judgement. The first is designed to ensure that an identifier can only be used in the construction of a type if it has already been declared in the environment. For example one can not deduce

$$
y:\left\langle x:(\text { nat })^{0}\right\rangle, x:(\text { nat })^{0} \vdash \text { Env }
$$

because the variable $x$ is used in the type associated with $y$ before being introduced. However if they are interchanged then this does constitute a valid environment:

$$
x:(\text { nat })^{0}, y:\left\langle x:(\text { nat })^{0}\right\rangle \vdash \text { Env }
$$

This emphasises the fact that our typing system will not have an interchange rule; in general being able to form a judgement of the form

$$
\Gamma, x: \tau, y: \tau^{\prime}, \Gamma^{\prime} \vdash \mathrm{J}
$$

will not necessarily imply

$$
\Gamma, y: \tau^{\prime}, x: \tau, \Gamma^{\prime} \vdash \mathrm{J}
$$

When constructing well-formed environments only types which are currently well-formed may be used. This is the purpose of the second form of judgement. So for example we can not deduce

$$
\Gamma, y:\left\langle y:(\text { nat })^{0}\right\rangle \vdash \text { Env }
$$

To do so we would need to be able to deduce

$$
\Gamma \vdash\left\langle y:(\text { nat })^{0}\right\rangle: \mathrm{tp}
$$

This in turn is not possible, basically because $y$ is not in the domain of $\Gamma$.

In the rules for $\Gamma \vdash \alpha: \operatorname{tp}$ one is only constrained to use identifiers which are already declared in the current environment. In (t-chan), the condition $S_{\mathrm{I}} \geq S_{0}$ is necessary to ensure that readers of a channel receive at most the capabilities given by a sender. There are only two novelties. In the formation rule for dependent types, $\left(\mathrm{t}-\mathrm{abs}_{N}\right)$ the bound variable $x$ is allowed in the construction of the result type $\rho$. Secondly the rule (t-proc) ensures a process always has a type $\Delta$ which does not exceed the current environment $\Gamma$.
Subtyping also plays a role in the formation of environments. For example we can not deduce

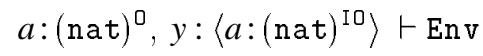

because the capability associated with $a$ when forming the type associated with $y$ is not a subtype of that associated with $a$ in the current environment. For no $\Gamma$ can we deduce

$$
\Gamma \vdash(\text { nat })^{0} \leq(\text { nat })^{\text {IO }}
$$

The rules for subtyping are a straightforward extension of those given in $[37,26,12]$, apart from the necessity to only use identifiers declared in the current environment. Function types are contravariant in their first arguments and covariant in their second, while, in (s-chan), channel types are covariant in the input capability and contravariant in the output. Again the only real novelty is the subtyping rule for process types, (s-proc); this means the ordering of process types is contravariant w.r.t. the ordering of [37, 26].

A series of consistency lemmas about this system of judgements follows. They are invariably deduced by induction on the derivations in the standard manner. Remember informally $X, Y, Z, \ldots$ denote variables with higher-order types, as opposed to channel types.

Lemma 3.1 (1) (Renaming) Suppose $u \notin \mathrm{fv}\left(\Gamma, v, \tau, \Gamma^{\prime}\right)$. Then $\Gamma, u: \tau, \Gamma^{\prime} \vdash \mathrm{J}$ implies $\Gamma, v: \tau, \Gamma^{\prime}\{v / u\} \vdash$ $\mathrm{J}\{v / u\}$.

(2) (Implied Judgement) $\Gamma, \Gamma^{\prime} \vdash \mathrm{J}$ implies $\Gamma \vdash$ Env and $\Gamma, u: \tau, \Gamma^{\prime} \vdash$ Env implies $\Gamma \vdash \tau:$ tp.

(3) (HO-bound Change) $\Gamma, X: \sigma_{H}, \Gamma^{\prime} \vdash \mathrm{J}$ and $\Gamma \vdash \sigma_{H}^{\prime}: \mathrm{tp}$ imply $\Gamma, X: \sigma_{H}^{\prime}, \Gamma^{\prime} \vdash \mathrm{J}$.

(4) (Weakening) Assume $\Gamma, x: \tau \vdash \operatorname{Env}$ and $u \notin \operatorname{dom}\left(\Gamma^{\prime}\right)$. Then $\Gamma, \Gamma^{\prime} \vdash \mathrm{J}$ implies $\Gamma, u: \tau, \Gamma^{\prime} \vdash \mathrm{J}$.

(5) (Multiple Weakening) Assume $\Gamma, \Gamma^{\prime \prime} \vdash$ Env and $\operatorname{dom}\left(\Gamma^{\prime}\right) \cap \operatorname{dom}\left(\Gamma^{\prime \prime}\right)=\emptyset$. Then $\Gamma, \Gamma^{\prime} \vdash \mathrm{J}$ implies $\Gamma, \Gamma^{\prime \prime}, \Gamma^{\prime} \vdash \mathrm{J}$.

(6) (Bound Weakening) Assume $\Gamma \vdash \tau^{\prime} \leq \tau$. Then $\Gamma, u: \tau, \Gamma^{\prime} \vdash \mathrm{J}$ implies $\Gamma, u: \tau^{\prime}, \Gamma^{\prime} \vdash \mathrm{J}$.

(7) (Implied Judgement) $\Gamma \vdash \alpha \leq \alpha^{\prime}$ implies $\Gamma \vdash \alpha$ : tp and $\Gamma \vdash \alpha^{\prime}:$ tp.

(8) (HO Narrowing) $\Gamma, X: \sigma_{H}, \Gamma^{\prime} \vdash \mathrm{J}$ implies $\Gamma, \Gamma^{\prime} \vdash \mathrm{J}$.

(9) (Exchange) Assume $\Gamma, u^{\prime}: \tau^{\prime} \vdash$ Env. Then we have $\Gamma, u: \tau, u^{\prime}: \tau^{\prime}, \Gamma^{\prime} \vdash \mathrm{J}$ implies $\Gamma, u^{\prime}: \tau^{\prime}, u: \tau, \Gamma^{\prime} \vdash \mathrm{J}$.

Note that in general we can not replace $X: \sigma_{H}$ with $u: \sigma$ in the statements (3) and (8) above since the channel $u$ may appear freely in $\Gamma^{\prime}$ and J. This underlines the major technical difference between our system and the system $F_{<:}$, [6];

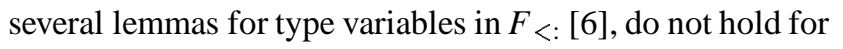
channels in our system.

We now turn our attention to the partial join operator $\sqcup$ which plays a crucial role in our definition of substitution,

Definition 3.2 (FBC, cf. [12, 37]) We say that a partial or$\operatorname{der}(\mathbf{S}, \sqsubset)$ is finite bounded complete (FBC) for every finite nonempty subset $S \leq \mathbf{S}$, if $S$ has a lower bound then $S$ has a greatest lower bound. 


\section{Well-formed Environment}

$$
\text { (e-nil) } \quad \emptyset \vdash \operatorname{Env} \quad \text { (e-val) } \frac{\Gamma \vdash \tau: \operatorname{tp} u \notin \operatorname{dom}(\Gamma)}{\Gamma, u: \tau \vdash \operatorname{Env}}
$$

\section{Well-formed Types}

$$
\begin{array}{llll}
\text { (t-base) } & \frac{\Gamma \vdash \text { Env }}{\Gamma \vdash \mathrm{T}, \perp, \sigma_{B}, \text { proc },[]: \mathrm{tp}} & (\mathrm{t}-\mathrm{sort}) \frac{\Gamma \vdash \tau_{i}: \mathrm{tp}}{\Gamma \vdash\left(\tau_{1}, \ldots, \tau_{n}\right): \mathrm{tp}} & (\mathrm{t}-\mathrm{chan}) \frac{\Gamma \vdash S_{\mathrm{I}}>S_{0}}{\Gamma \vdash\left\langle S_{\mathrm{I}}, S_{0}\right\rangle: \mathrm{tp}} \\
\left(\mathrm{t}-\mathrm{abs} H_{H}\right) \frac{\Gamma \vdash \sigma_{H}: \mathrm{tp}, \rho: \mathrm{tp}}{\Gamma \vdash \sigma_{H} \rightarrow \rho: \mathrm{tp}} & \left(\mathrm{t}-\mathrm{abs}_{N}\right) \frac{\Gamma, x: \sigma \vdash \rho: \mathrm{tp}}{\Gamma \vdash(x: \sigma) \rightarrow \rho: \mathrm{tp}} & (\mathrm{t}-\mathrm{proc}) \frac{\forall u \in \operatorname{dom}(\Delta) \cdot \Gamma \vdash \Gamma(u) \leq \Delta(u)}{\Gamma \vdash[\Delta]: \mathrm{tp}}
\end{array}
$$

Subtyping

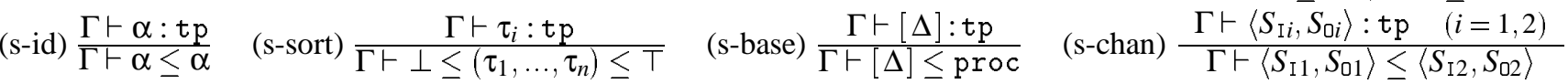

$$
\begin{aligned}
& \left(\mathrm{s}-\mathrm{abs}_{H}\right) \quad\left(\mathrm{s}-\mathrm{abs}_{N}\right) \\
& \frac{\Gamma \vdash \sigma_{H}^{\prime} \leq \sigma_{H}, \quad \rho \leq \rho^{\prime}}{\Gamma \vdash \sigma_{H} \rightarrow \rho \leq \sigma_{H}^{\prime} \rightarrow \rho^{\prime}} \quad \frac{\Gamma \vdash \sigma_{2} \leq \sigma_{1} \quad \Gamma, x: \sigma_{1} \vdash \rho_{1} \leq \rho_{2}}{\Gamma \vdash\left(x: \sigma_{1}\right) \rightarrow \rho_{1} \leq\left(x: \sigma_{2}\right) \rightarrow \rho_{2}} \quad \frac{\forall u \in \operatorname{dom}\left(\Delta_{2}\right) . \Gamma \vdash \Delta_{1}(u) \leq \Delta_{2}(u)}{\Gamma \vdash\left[\Delta_{2}\right] \leq\left[\Delta_{1}\right]}
\end{aligned}
$$

Figure 6. Well-formed Types and Subtyping

Proposition 3.3 Under an arbitrary well-formed environment, the subtyping relation over types is a partial order and finite bounded complete.

The proof of this proposition, available in [38], is constructive; we give an inductive definition of the required partial meet operation $\sqcap$; this requires a simultaneous definition of a partial join operation $\sqcup$, the operation we have already used above.

The next Lemma will be important in the proof of Subject Reduction. It shows that, under certain circumstances, a variable can be replaced by an identifier throughout a judgement, although this replacement may also change the environment of the judgement.

Lemma 3.4 (Name substitution) Suppose $\Gamma \vdash \Gamma(u) \leq \sigma$. Then $\Gamma, x: \sigma, \Gamma^{\prime} \vdash \mathrm{J}$ implies $\Gamma, \Gamma^{\prime}\{u / x\} \vdash \mathrm{J}\{u / x\}$.

Finally we conclude this section with the following property, which relates to the construction of an efficient algorithm of the typing system (cf. [26, 37]).

Proposition 3.5 (Decidability) $\Gamma \vdash J$ is decidable. Moreover, if $\Gamma \vdash \alpha_{i}: \operatorname{tp}(i=1,2)$, then $\Gamma \vdash \alpha_{1} \sqcup \alpha_{2}: \operatorname{tp}$ and $\Gamma \vdash \alpha_{1} \sqcap \alpha_{2}:$ tp are decidable.

\subsection{Type Inference}

The typing system is given in Figure 7. The judgements are of the form:

$$
\Gamma \vdash P: \alpha \quad \text { a term } P \text { has a type } \alpha \text { under } \Gamma
$$

For convenience the inference rules in Figure 7 are divided into three groups. The first, (Common), are elementary, although the subsumption rules $\left(\mathrm{SUB}_{H}\right)$ and $\left(\mathrm{SUB}_{N}\right)$ will play a major role in type inferences. The second, (Function), are inherited from typing systems for the polymorphic $\lambda$ calculus. Here we have two forms of functional types, each with its introduction and elimination rules. The novelty occurs with abstraction over channel variables. Intuitively if a term $P$ has a type $\rho$, then a channel abstraction $\lambda(x: \sigma) P$ is a function which becomes $P\{a / x\}$ when it is applied to a name $a$ with a type $\sigma$. Therefore, we will bind free occurrences of $x$ in $\rho$ in the abstraction rule $\left(\mathrm{ABS}_{N}\right)$. The corresponding elimination $\left(\mathrm{APP}_{N}\right)$ allows dynamic channel instantiation into types during $\beta$-reduction. If a term $P$ has a type $(x: \sigma) \rightarrow \rho$, we can apply it to a name $a$ whose type is less than $\sigma$ to $P$. Then $a$ is substituted for $x$ in $\rho$ in $\left(\operatorname{APP}_{N}\right)$.

The final group, (Process), are based on the IO-Typing systems from [26, 12, 37]. However many of the rules are sufficient novel to warrant detailed explanation.

The empty rule, (NIL): A process type $[\Delta]$ of represents an upper bound on the interface or interaction points of a process. Since an empty process $\mathbf{0}$ has no interaction point, under any well-formed environment $\Gamma$ it is typed as:

$$
\Gamma \vdash \mathbf{0}:[]
$$

The parallel rule, (PAR): To infer $\Gamma \vdash P_{1} \mid P_{2}: \pi$ it is sufficient to infer $\Gamma \vdash P_{1}: \pi$ and $\Gamma \vdash P_{2}: \pi$ individually. However, in the presence of subsumption, there is a more informative derived version of this rule, which will be frequently used:

$$
\frac{\Gamma \vdash P_{1}: \pi_{1}, \Gamma \vdash P_{2}: \pi_{2}}{\Gamma \vdash P_{1} \mid P_{2}: \pi_{1} \sqcup \pi_{2}}
$$

A meta-result of our system ensures that, relative to a particular environment, the join of process types always exists.

The output rule, (OUT): Under what circumstances can we conclude $\Gamma \vdash a !\langle V\rangle P: \pi$ ? We require the following:

- The residual $P$ should have the required type, $\Gamma \vdash P: \pi$ 


\begin{tabular}{|c|c|c|c|c|c|}
\hline \multirow[t]{2}{*}{ (Common) } & (VAL) & \multicolumn{2}{|l|}{$\frac{\vdash \Gamma, u: \tau, \Gamma^{\prime}: \text { Env }}{\Gamma, u: \tau, \Gamma^{\prime} \vdash u: \tau}$} & $(\mathrm{CON})$ & $\frac{\vdash \Gamma: \text { Env }}{\Gamma \vdash 1: \text { nat }} \quad$ etc. \\
\hline & $\left(\mathrm{SuB}_{H}\right)$ & \multicolumn{2}{|l|}{$\frac{\Gamma \vdash P: \rho \quad \Gamma \vdash \rho \leq \rho^{\prime}}{\Gamma \vdash P: \rho^{\prime}}$} & $\left(\mathrm{SUB}_{N}\right)$ & $\frac{\Gamma \vdash u: \sigma \quad \Gamma \vdash \sigma<\sigma^{\prime}}{\Gamma \vdash u: \sigma^{\prime}}$ \\
\hline \multirow[t]{2}{*}{ (Function) } & $\left(\mathrm{ABS}_{H}\right)$ & \multirow{2}{*}{\multicolumn{2}{|c|}{$\begin{array}{c}\frac{\Gamma, X: \sigma_{H} \vdash P: \rho}{\Gamma \vdash \lambda\left(X: \sigma_{H}\right) P: \sigma_{H} \rightarrow \rho} \\
\frac{\Gamma, x: \sigma \vdash P: \rho}{\Gamma \vdash \lambda(x: \sigma) P:(x: \sigma) \rightarrow \rho}\end{array}$}} & $\left(\mathrm{APP}_{H}\right)$ & $\frac{\Gamma \vdash P: \sigma_{H} \rightarrow \rho \quad \Gamma \vdash Q: \sigma_{H}}{\Gamma \vdash P Q: \rho}$ \\
\hline & $\left(\mathrm{ABS}_{N}\right)$ & & & $\left(\mathrm{APP}_{N}\right)$ & $\frac{\Gamma \vdash P:(x: \sigma) \rightarrow \rho \quad \Gamma \vdash u: \sigma}{\Gamma \vdash P u: \rho\{u / x\}}$ \\
\hline \multirow[t]{2}{*}{ (Process) } & (NIL) & $\begin{array}{l}\vdash \Gamma: \text { Env } \\
\Gamma \vdash \mathbf{0}:[]\end{array}$ & $\frac{\Gamma \vdash P_{1,2}: \pi}{\Gamma \vdash P_{1} \mid P_{2}: \pi}$ & (REP) & $\frac{\Gamma \vdash P: \pi}{\Gamma \vdash * P: \pi} \quad$ (RES) $\frac{\Gamma, a: \sigma \vdash P: \pi}{\Gamma \vdash(\vee a: \sigma) P: \pi / a}$ \\
\hline & (OUT) & $\begin{array}{l}\pi \vdash_{\Gamma} u:\left(\tau_{1}, \ldots, \tau_{n}\right)^{0} \\
\Gamma \vdash V_{i}: \tau_{i} \quad \tau_{i}=\sigma_{i} \Rightarrow \pi \\
\Gamma \vdash u !\left\langle V_{1}, \ldots, V_{n}\right\rangle P: \pi\end{array}$ & $\begin{array}{l}\Gamma \vdash P: \pi \\
\vdash_{\Gamma} V_{i}: \sigma_{i} \\
\end{array}$ & (IN) & $\begin{array}{l}\pi \vdash \Gamma u:\left(\tau_{1}, \ldots, \tau_{n}\right)^{\mathrm{I}} \\
\Gamma, x_{1}: \tau_{1}, \ldots, x_{n}: \tau_{n} \vdash P: \pi, x_{1}: \tau_{1}, \ldots, x_{n}: \tau_{n} \\
\Gamma \vdash u ?\left(x_{1}: \tau_{1}, \ldots, x_{n}: \tau_{n}\right) P: \pi\end{array}$ \\
\hline
\end{tabular}

In (OUT) and (IN), $\pi \vdash_{\Gamma} u: \sigma$ means $\Gamma \vdash[u: \sigma] \leq \pi$.

In (IN), $\pi, x: \tau$ is defined as: (1) $\pi, x: \sigma \stackrel{\text { def }}{=} \pi \sqcup[x: \sigma]$, and (2) $\pi, x: \sigma_{H} \stackrel{\text { def }}{=} \pi$.

Figure 7. Typing System for $\lambda \pi_{\mathrm{v}}$

- The value $V$ should have a type appropriate to the channel $a$. That is, there should be some value type $\tau$ such that $\Gamma \vdash V: \tau$ and

- the channel $a$ should have the output capability at the type $\tau$. However this capability on $a$ should be available from the overall interface of the process, $\pi$. This can be represented by the judgement $\Gamma \vdash\left[a:(\tau)^{0}\right] \leq \pi$.

However there may be a further requirement. If the value being output is actually a channel, say $b$ with a type $\tau=\sigma$, then the capability being exported must also be available from the process interface $\Gamma \vdash[b: \sigma] \leq \pi$.

The general statement of the rule, for multiple output values, is given in Figure 7; it uses the notation defined in Figure 7

$$
\pi \vdash_{\Gamma} u: \sigma
$$

to mean that, relative to the environment $\Gamma$ the interface, or process type, $\pi$ can provide at least the capability $\sigma$ at $u$.

As an example let $\Delta_{a b}$ be the environment which maps $b$ to the type (int) ${ }^{0}$ and $a$ to the type $\left\langle\Delta_{b}\right\rangle^{{ }^{\mathrm{T}}}$, allowing it to transmit thunked values of type $\Delta_{b}$, which maps $b$ to the same type (int) ${ }^{0}$. Then with the output rule, together with (NIL), we can establish

$$
\Delta_{a b} \vdash b !\langle 1\rangle \mathbf{0}:\left[\Delta_{b}\right]
$$

and therefore

$$
\Delta_{a b} \vdash a !\langle b !\langle 1\rangle \mathbf{0}\rangle \mathbf{0}:\left[a:\left\langle\Delta_{b}\right\rangle^{0}\right]
$$

The input rule, (IN): The rule for prefixing is a straightforward generalisation of that in [37]:

$$
\frac{\pi \vdash \Gamma a:(\tau)^{\mathrm{I}} \quad \Gamma, x: \tau \vdash P: \pi, x: \tau}{\Gamma \vdash a ?(x: \tau) P: \pi}
$$

$$
\begin{aligned}
\mathrm{T} / a=\mathrm{T}, \perp / a=\perp, & \sigma_{B} / a=\sigma_{B}, \text { proc } / a=\text { proc } \\
\left\langle S_{\mathrm{I}}, S_{0}\right\rangle / a & =\left\langle S_{\mathrm{I}} / a, S_{0} / a\right\rangle \\
\left(\tau_{1}, \ldots, \tau_{n}\right) / a & =\left(\tau_{1} / a, \ldots, \tau_{n} / a\right) \\
\left(\sigma_{H} \rightarrow \rho\right) / a & =\sigma_{H} / a \rightarrow \rho / a \\
((x: \sigma) \rightarrow \rho) / a & =(x: \sigma / a) \rightarrow \rho / a \\
{[\Delta] / a } & =[\{u:(\sigma / a) \mid u: \sigma \in \Delta \wedge u \neq a\}]
\end{aligned}
$$

Figure 8. Name Erasing on Types

To deduce that the process $a ?(x: \tau) P$ has the interface $\pi$ we need to establish two facts:

- The interface $\pi$ can provide the correct capability for the channel $a$; that is $\pi \vdash_{\Gamma} a:(\tau)^{\mathrm{I}}$.

- The residual $P$, having input a value for the variable $x$, has the augmented interface $\pi, x: \tau$; however this can be established in the environment $\Gamma$ augmented by $x$; that is $\Gamma, x: \tau \vdash P: \pi, x: \tau$.

Here we are using a notation " $\pi, x: \tau$ " defined in Figure 7. Note " $\pi, x: \tau$ " denotes " $\pi$ " if $\tau$ is not a channel type. In (IN), by the first sequence in the antecedent and (Implied judgement), Lemma 3.1, we know $\pi$ is well-formed under $\Gamma$. Hence automatically $x$ does not occur in $\pi$. From this, if $\pi$ takes the form $[\Delta]$ for some $\Delta$, then $x \notin \mathrm{fv}([\Delta])$, and $P$ has a type $[\Delta], x: \sigma \stackrel{\text { def }}{=}[\Delta, x: \sigma]$ in the second assumption.

As an example let $\Delta_{c}$ be the environment which maps $c$ to the capability $\left((\text { int })^{0}\right)^{\mathrm{IO}}$. Then one can easily check that

$$
\Delta_{c} \vdash c ?\left(z:(\text { int })^{0}\right) z !\langle 1\rangle:\left[\Delta_{c}\right]
$$

It may seem strange that this process has been typed to have at most a capability on the channel $c$; obviously when it re- 
ceives an input on $c$ it will immediately gain some other capability. But this input will be sent by some other process, in the presence of which the interface will be increased appropriately. For example let $\Delta_{c d}$ be the extension of $\Delta_{c}$ which maps $d$ to the output capability, (int $)^{0}$. Then we have

$$
\Delta_{c d} \vdash c !\langle d\rangle:\left[\Delta_{c d}\right]
$$

Now we can use the rule (PAR), or rather its derived variant, (together with a version of Multiple Weakening) to deduce

$$
\Delta_{c d} \vdash\left(c ?\left(z:(\text { int })^{0}\right) z !\langle 1\rangle \mid c !\langle d\rangle\right):\left[\Delta_{c d}\right]
$$

The restriction rule, (RES): The restriction operator $(\mathrm{v} a)$ - reduces the interface of a process. For example in an appropriate environment the process $a !\langle 1\rangle$ can be assigned the process type, or interface, $\left[a:(\text { nat })^{0}\right]$. When we restrict the channel $a$, to obtain the process $(v a) a !\langle 1\rangle$, all $a$ capabilities will be removed from the interface; the restricted process has the empty interface []. The general rule is formulated as (RES) where $\pi / a$ denotes the result of erasing all occurrences of $a$ from $\pi$. This erasure operator on types is defined formally in Figure 8. For example, $\left[a:(\text { nat })^{0}\right] / a=[]$, and $\left[b:\left\langle a:(\text { nat })^{0}\right\rangle^{0}\right] / a=\left[b:\langle\rangle^{0}\right]$. Hence, in appropriate environments, ( $\mathrm{v} a) a !\langle 1\rangle$ has a type [] and $(v a) b !\langle a !\langle 1\rangle\rangle$ has a type $\left[b:\langle\rangle^{0}\right]$.

Example 3.6 We revisit Example 2.2. First the successor service is annotated as:

$$
\operatorname{Succ}(a) \Longleftarrow * a ?\left(y: \text { int }, z:(\text { int })^{0}\right) z !\langle\operatorname{succ}(y)\rangle
$$

where the types ensure that this process only receives the output capability on the return channel $z$. Let $\Delta_{r}$, be an environment defining these return channels; in this case it maps $r_{A}, r_{1 B}$ and $r_{2 B}$ to the same type (int) ${ }^{0}$. Let $\sigma_{s}$ be a type $\left(\text { int },(\text { int })^{0}\right)^{0}$ and $\tau_{\mathrm{sc}}$ be a type for scripts:

$$
\left(s: \sigma_{s}\right) \rightarrow\left(p: \sigma_{s}\right) \rightarrow\left[s: \sigma_{s}, p: \sigma_{s}, \Delta_{r}\right]
$$

So these are abstractions which, when applied to appropriate names, generate processes which can at most use those names for output, together with the return channels, also for output only. Using subsumption we can form the judgement

$$
s: \sigma_{s}, p: \sigma_{s}, \Delta_{r} \vdash P_{A}:\left[s: \sigma_{s}, p: \sigma_{s}, \Delta_{r}\right]
$$

where $P_{A}$ denotes the body of the script sent by $\mathbf{C}_{A}$, namely $(v c) s !\langle k, c\rangle c ?(z) s !\langle z, c\rangle \mathrm{Fw}\left(c r_{A}\right)$. By channel abstraction we therefore have

$$
\Delta_{r} \vdash \lambda\left(s: \sigma_{s}, p: \sigma_{s}\right) \cdot P_{A}: \tau_{\mathrm{sc}}
$$

That is the value sent by the client is indeed typed as a script.

Now let $\Delta_{\mathrm{cl}}$ denote the environment $\left\{\right.$ req : $\left.\left(\tau_{\mathrm{sc}}\right)^{\mathrm{D}}, \Delta_{r}\right\}$. Then we can form the judgement

$$
\Delta_{\mathrm{cl}} \vdash \mathbf{C}_{A}(\mathrm{req}):\left[\Delta_{\mathrm{cl}}\right]
$$

and a similar judgement can be made for $\mathbf{C}_{B}$.

This judgement gives detailed information about the resources known to the clients. For example it says that the clients do not need to know the locations of the actual interfaces of the various services; indeed it only needs to know that of the server, req, together with the return channels.
Typing the server is slightly different. Here we need to let $\Delta_{\text {serv }}$ be $\left\{\right.$ req $\left.:\left(\tau_{\mathrm{sc}}\right)^{\mathrm{I}}, \Delta_{r}, s: \sigma_{s}, p: \sigma_{s}\right\}$. Then we can check that

$$
\Delta_{\text {serv }} \vdash \operatorname{Serv}_{I}(\text { req, } s, p):\left[\Delta_{\text {serv }}\right]
$$

Thus the server requires knowledge of the locations of the service points, but needs only to be able to send data to them. It also only sends capabilities on the return channels. Note also that if we only have a constant process type proc, as in the previous typing systems for the process calculi $[3,37,26]$, then the interface server could input any function $\lambda s . \lambda$ p. $Q$, where $Q$ an arbitrary process via "req"; such incoming code may harm local resources.

\section{Type Soundness}

\subsection{Subject Reduction}

The results in Lemma 3.1 have natural generalisations to our typing system, $\Gamma \vdash P: \alpha$. The details are available in [38], but here we show one instance, Channel Narrowing.

Lemma 4.1 Assume $a \notin \mathrm{fn}(P)$. Then $\Gamma, a: \sigma, \Gamma^{\prime} \vdash \mathrm{J}: \alpha$ implies $\Gamma, \Gamma^{\prime} / a \vdash \mathrm{J}: \alpha / a$, and $\Gamma, a: \sigma, \Gamma^{\prime} \vdash P: \alpha$ implies $\Gamma, \Gamma^{\prime} / a \vdash P: \alpha / a$.

The following result which states, informally, that welltypedness is preserved by substitution of appropriate values for variables, is the key result underlying Subject Reduction. This also guarantees that substitution, which uses $\sqcup$ in its definition, is always defined when applied to well-typed terms.

Lemma 4.2 (Substitution Lemma) Assume $\Gamma \vdash V: \tau$. Then $\Gamma, x: \tau, \Gamma^{\prime} \vdash P: \alpha$ implies $\Gamma, \Gamma^{\prime}\{V / x\} \vdash P\{V / x\}: \alpha\{V / x\}$.

\section{Theorem 4.3 (Subject Reduction)}

- $\Gamma \vdash P: \pi$ and $P \equiv P^{\prime}$ imply $\Gamma \vdash P^{\prime}: \pi$.

- If $\Gamma \vdash P: \rho$ and $P \longrightarrow P^{\prime}$, then $\Gamma \vdash P^{\prime}: \rho$.

\subsection{Type Safety}

Our typing system is an extension of that for the $\lambda$-calculus from [9] and that for the $\pi$-calculus from [26]; consequently it guarantees the absence of the typical run-time errors associated with these languages. Rather than duplicate the formulation of these kinds of errors, which involves the development complicated tagging notation, here we concentrate on the novel run-time type errors which our typing system can catch.

Intuitively $\Gamma \vdash P: \pi$ should mean that, assuming the environment $\Gamma$, the process $P$ satisfies the interface $\pi$. If $\pi$ is the undifferentiated type proc then, viewed as an interface, it provides no information. However if it has the form $[\Delta]$ this means that $P$ can use at most the resources mentioned in $\Delta$; moreover these resources can only be used according to the capabilities they are assigned in $\Delta$. A simple formalisation of this intuitive idea is given in Figure 9, using a unary predicate $P \stackrel{\Gamma, \pi}{\longrightarrow}$ er . The first two clauses are the most 


$$
\begin{aligned}
& a ?\left(x_{1}: \tau_{1}, \ldots, x_{n}: \tau_{n}\right) P \stackrel{\Gamma, \pi}{\longrightarrow}_{e r} \quad \text { if } \Gamma \nvdash\left[a:\left(\tau_{1}, \ldots, \tau_{n}\right)^{\mathrm{I}}\right] \leq \pi . \\
& a !\left\langle V_{1}, \ldots, V_{n}\right\rangle P \stackrel{\Gamma, \pi_{e r}}{\longrightarrow} \quad \text { if no } \tau_{i} \text { with } \Gamma \vdash V_{i}: \tau_{i} \\
& \text { s.t. } \Gamma \vdash\left[a:\left(\tau_{1}, \ldots, \tau_{n}\right)^{\mathrm{D}}\right] \leq \pi .
\end{aligned}
$$

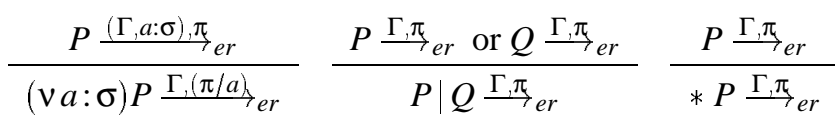

Figure 9. Run-time errors

significant. The first says that, relative to $\Gamma, P$ violates the interface $\pi$ if it can input on the channel $a$ but the interface $\pi$ does not assign any input capability to $a$; the second is similar, but for output. Combining these rules, we can also derive the following communication runtime error between input and output:

$$
a ?\left(x_{1}: \tau_{1}, \ldots, x_{n}: \tau_{n}\right) P \mid a !\left\langle V_{1}, \ldots, V_{n}\right\rangle Q \stackrel{\Gamma, \pi}{\longrightarrow} \text { er }
$$

if there is no $\tau_{i}^{\prime}$ such that $\Gamma \vdash \tau_{i}^{\prime} \leq \tau_{i}$ and $\Gamma \vdash V_{i}: \tau_{i}^{\prime}$. The meaning of the above error is easily understood when we consider the following example:

$$
a ?(x:\langle\Delta\rangle) P \mid a !\langle R\rangle Q \stackrel{\Gamma, \pi}{\rightarrow}_{e r} \quad \text { if } \Gamma \nvdash R:[\Delta] .
$$

This says if the input process gets the process $R$ which does not conform the interface " $\Delta$ ", then a runtime error occurs.

Theorem 4.4 (Type Safety) If $\Gamma \vdash P: \pi$ then $P \underset{\mathrm{F}, \pi}{\mathrm{r}}$.

\section{Typed Behavioural Semantics}

Types constrain the behaviour of processes and their environments and consequently have an impact on when their behaviour should be deemed to be equivalent. Typed behavioural equivalences have already been investigated for various process calculi in papers such as $[19,14,26,27,36]$. We contend that the existence of the fine-grained process types facilitates the development of typed behavioural theories; more importantly they enable us to state and prove general theorems about these equivalences which are extremely useful for establishing identities. Due to space limitations in this section we merely outline our results in this area.

A family of relations $\mathbf{R}$ over process terms, ranged over by closed type environments and process types, is said to be a typed relation if $P_{1} \mathbf{R}_{\pi}^{\Gamma} P_{2}$, implies $\Gamma \vdash P_{1}: \pi$ and $\Gamma \vdash P_{2}: \pi$. Note that the relation is parameterised by not only environment $\Gamma$ but also interface $\pi$. The following definition uses the notation from $[17,26,36]$.

Definition 5.1 Let $\approx \pi$ denote the largest typed relation which:

- is a typed congruence

- is closed under reductions: whenever $P \mathbf{R}_{\pi}^{\Gamma} Q, P \rightarrow P^{\prime}$ implies, for some $Q^{\prime}, Q \rightarrow Q^{\prime}$ and $P^{\prime} \mathbf{R}_{\pi}^{\Gamma} Q^{\prime}$.
- satisfies $P_{1} \mathbf{R}_{\pi}^{\Gamma} P_{2}$ implies $P_{1} \Downarrow_{a^{\mathrm{I}}} \Leftrightarrow P_{2} \Downarrow_{a^{\mathrm{I}}}$ and $P_{1} \Downarrow_{a^{0}} \Leftrightarrow P_{2} \Downarrow_{a^{0}}$ where $P \Downarrow_{a^{\mathrm{I}}} \stackrel{\text { def }}{\Leftrightarrow} \exists P^{\prime} . P \rightarrow P^{\prime} \wedge P^{\prime} \equiv$ $(\vee \tilde{c})\left(a ?(x) R \mid R^{\prime}\right)$ with $a \notin\{\tilde{c}\}$. Similarly for $P \Downarrow_{a^{0}}$.

Let $\sim \pi$ denote the corresponding strong relation.

We can establish expected identities such as:

Proposition 5.2 (1) (Garbage collection) $P \approx_{[]}^{\Gamma} \mathbf{0}$.

(2) (Beta-reduction) $(\lambda x . P) V \approx \pi P\{V / X\}$.

Note (1) is a more general garbage collection law than the standard one defined with the condition $\mathrm{fn}(P)=\emptyset$; for example, we can prove $(v a)(a ?(X) \mathbf{0} \mid a !\langle b ?(Y) P\rangle)$ is equivalent to $\mathbf{0 .}$

Next we show our fine-grained types also allow us to give a simple, and clean, definition of triggers which have proven to be important in the theory of the higher-order processes, as well as the $\pi$-calculus [26, 29].

Definition 5.3 We say $a$ is (only) used as a trigger in $P$ under $\Gamma$ if there exists $\Delta$ such that $\Gamma \vdash P:[\Delta]$, and if $a: \sigma \in \Delta$, then $\sigma=S^{0}$.

Note that the above definition is considerably simpler than that given in [26] (c.f. Definition 5.3.1, pp.433).

Proposition 5.4 (Higher-order replication) Assume $a$ is only used as a trigger in $P, Q$ and $R$ under $\Gamma$. Then:

$$
\begin{aligned}
& (\mathrm{v} a: \sigma)\left(P|Q| * a ?\left(x_{1}: \tau_{1}, \ldots, x_{n}: \tau_{n}\right) \cdot R\right) \\
& \sim \Gamma_{\pi}(\mathrm{v} a: \sigma)\left(P \mid * a ?\left(x_{1}: \tau_{1}, \ldots, x_{n}: \tau_{n}\right) \cdot R\right) \\
& \mid(\mathrm{v} a: \sigma)\left(Q \mid * a ?\left(x_{1}: \tau_{1}, \ldots, x_{n}: \tau_{n}\right) \cdot R\right)
\end{aligned}
$$

Again this is a non-trivial generalisation of the corresponding result in [29], Theorem 4.3.3; there " $a$ " is only allowed to appear syntactically as output subjects. The following forwarder equality is one useful extension from [16] to the higher-order setting.

Proposition 5.5 (Forwarder [16]) Assume $a$ is used as a trigger in $P, b \neq a$ and $\mathrm{F} w$ is the forwarder defined in Section 1. Then: $(a: \sigma)(P \mid \mathrm{F} w(a b)) \approx \pi P\{b / a\}$.

Finally we illustrate the usefulness of these general identities by applying them to the analysis of the system discussed in Example 3.6. Let Sys denote:

$(v$ req, $s, p)\left(\mathbf{C}_{A}(\right.$ req $) \mid \mathbf{C}_{B}($ req $) \mid \operatorname{Serv}_{I}($ req, $\left.s, p)|\operatorname{Succ}(s)| \operatorname{Pred}(p)\right)$ Intuitively in this closed system the two clients make three specific requests of the distributed server. So we have:

$$
\begin{array}{ll}
\text { Sys } & \\
\sim_{\pi} & (v \text { req, } s, p)\left(\mathbf{C}_{A}(\text { req })\left|\operatorname{Serv}_{I}(\operatorname{req}, s, p)\right| \operatorname{Succ}(s) \mid \operatorname{Pred}(p)\right) \\
& \mid(v \text { req, } s, p)\left(\mathbf{C}_{B}(\text { req })\left|\operatorname{Serv}_{I}(\operatorname{req}, s, p)\right| \operatorname{Succ}(s) \mid \operatorname{Pred}(p)\right) \\
\approx_{\pi} & r_{A} !\langle k+2\rangle\left|r_{B 1} !\langle n+1\rangle\right| r_{B 2} !\langle m-1\rangle
\end{array}
$$

The first equation is an instance of Proposition 5.4 (since by $\tau_{\mathrm{sc}}$ which annotates variable $X$ in $\operatorname{Serv}_{I}($ req, $s, p)$, we can check $s$ and $p$ are used as a trigger in the server). Note that $s$ and $p$ appear as values, hence they may be used both as output subjects and objects in the interface server; therefore 
our generalised version of this theorem is required. The second equation requires Proposition 5.2.

Further investigation of our typed equivalences is an interesting research topic, particularly in its application to the refinement of the context equality of [29].

\section{Conclusion}

This Section concludes the paper with extensions, further issues and related work. For more details, see [38].

Distributed Higher-order $\pi$-calculus Type Safety Theorem means that our typing system can be used to ensure various kinds of host security; that is, protecting hosts from untrusted imported code. In the full version of this paper, [38], we discuss this issue more explicitly, by extending our typing system to the distributed version of $\lambda \pi_{\mathrm{v}}$, given in [37]. Once more the expressiveness of our fine-grained types means that, for example, channel locality, [37], can easily be enforced; specifically there is no requirement to annotate higher-order values as being sendable. A similar problem appears with type systems for the $\lambda$-calculus involving arrow and reference types, [24]. We hope that an extension of our scheme to higher-order functional shared variables (i.e. passing environments as com types) will also be useful in this setting.

More type constructors One beneficial point of our typing system is that it is relatively straightforward to extend our set of types with many of the standard constructs from the literature for both the $\lambda$-calculus and the $\pi$-calculus; these include recursive types $[4,21,33,26]$, record types [9, 32], polymorphic types [6, 27], linear/affine types $[19,5,13,36]$, and dynamic types [2, 28]. An extension of our capability based typing systems to more advanced distributed primitives, especially to constructs involving security $[1,11,15,35]$ would be more challenging.

Type limitations One limitation of our typing system is that, while name variables in types can be abstracted by channel dependency types $(x: \sigma) \rightarrow \rho$ of the channel $\lambda$ abstraction $\lambda(x: \sigma) P$, a similar abstraction is not allowed when we bound name variables by input prefix $a$ ? $(x: \sigma) P$. The result is that there is a loss of information in many of the types we can assign to processes. A typical example is the process $a ?(x) b !\langle x !\langle v\rangle\rangle$. In the current system this can only be assigned a process type in which $b$ has the capability to output values of the undifferentiated type $\langle$ proc $\rangle$.

Clearly some form of channel abstraction would be needed to give a more informative type but it is difficult to see how this might be formulated. One problem here is that, unlike $\beta$-application, value reception is nondeterministic. In the composed term

$$
a ?(x) b !\langle x !\langle v\rangle\rangle|a !\langle c\rangle| a !\langle d\rangle
$$

the particular channel, $c$ or $d$ which is bound to $x$ depends on which message is delivered to the waiting process. Indeed the residual, after receiving an input, may take one of the (incomparable) types $\left[b:\langle c: \sigma\rangle^{0}\right]$ or $\left[b:\langle d: \sigma\rangle^{0}\right]$.
There is a similar loss of information in typing restricted processes, $(v a) P$. For example the process $(v a) b !\langle a !\langle 1\rangle\rangle$ can be assigned, in an appropriate environment, the type $\left[b:\langle\rangle^{0}\right]$ which intuitively says that $b$ can output a (thunked) process which has the empty interface. This type is of limited interest when used in context. For example consider

$$
(v a) b !\langle a !\langle 1\rangle\rangle \mid b ?(X: \tau) \text { run } X
$$

Here essentially the only the possibility for $\tau$ is the type $\langle$ proc $\rangle$. But we should be able to say that $b$ can output a (thunked) process which contains some unknown channel name of type (nat) ${ }^{\mathrm{D}}$, and the input type associated with $b$ should be able to accommodate such constraints. Some form of existential quantification over types may be appropriate but integrating such a construct into the type language is also a non-trivial task.

Related work We have already made reference to the extensive literature on typing for the $\pi$-calculus and related processes. In developing our fine-grained type system we have been guided by the polymorphic $\lambda$-calculus $[9,6]$, where type variables play an important role; as with our channel names they may appear, and be bound, both in terms and types. However there is an essential technical difference: channel instantiation in our system can result in dynamic changes to the types annotating a term. Channels are exchanged as values between processes but they also appear as interaction points in the types of processes. On the other hand, type variables of the polymorphic $\lambda$ calculus are instantiated by types (say int) whereas in our case channel variables occurring in types are instantiated by channels, not by types. This feature necessitated the development of new concepts of well-formed type, subtyping, well-formed substitution, etc., independent of those developed in the context of the $\lambda$-calculus.

Pierce and Sangiorgi [27] recently proposed a polymorphic $\pi$-calculus and used a refined typed behavioural equivalence to reason about concurrent abstract data types. Since their polymorphic types are based on those of the polymorphic $\lambda$-calculus (that is they abstract over type variables via the operator $\exists$ ), they are quite different from ours. In particular they do not address the issue of assigning fine-grained types to processes.

For sequential computations, Tofte and Talpin have developed the effect typing system [31], and Tang and Jouvelot developed its subtyping system [30]. This was recently applied to Facile by Kirli [18]. ${ }^{1}$ One may think our dependency types correspond to her region polymorphism. However, again her typing system is different from ours since she adds the original effect system to the functional types; hence all process has a constant type Unit and channels cannot carry nested effects. More precisely, the effect types in $[31,30]$ are used to represent the region allocation or effects of values during $\beta$-reduction, while our process types are used to represent interaction effects between con-

\footnotetext{
${ }^{1}$ Kobayashi, Nakade and Yonezawa also applied the effect typing system to a concurrent logic programming in [20].
} 
current processes. Hence an integration of the effect typing system of the $\lambda$-calculus and the IO-subtyping system of the $\pi$-calculus would have difficulty in expressing the kind of constraints guaranteed by our typing system.

De Nicola, Ferrari and Pugliese studied a subtyping system for a language based on Linda [7], and showed that it is used to control the mobility of agents. In their language, each located process is equipped with different capabilities (read, input, our, eval and newloc) rather than the unique process type, which is similar to our framework. However their calculus is based on CCS rather than the $\pi$-calculus and our form of process types based on IO-subtyping and $\lambda$-subtyping are not considered in their formulation.

Acknowledgements We thank Kohei Honda, Dilsun Kirli, Naoki Kobayashi, Didier Rémy, Vasco Vasconcelos, and anonymous referees for their comments and discussions.

\section{References}

[1] Abadi, M., Secrecy by Typing in Security Protocols, TACS'97, LNCS 1281, pp.611-638, Springer-Verlag, 1997.

[2] Abadi, M., Cardelli, L., Pierce, B. and Plotkin, G., Dynamic Typing in a Statically Typed Language. TOPLAS, 13(2), pp.237-268, 1991.

[3] Amadio, R., Translating Core Facile, ECRC Research Report 944-3, 1994.

[4] Amadio, R. and Cardelli, L., Subtyping Recursive Types, TOPLAS, 15(4), pp.575-631, 1993.

[5] Cardelli, L. and Gordon, A., Typed Mobile Ambients, POPL'99, pp.79-92, ACM Press, 1999.

[6] Cardelli, L., Martini, S., Mitchell, J. and Scedrov, A., An extension of system F with subtyping, Info. \& Comp., 109(1):456, February 1994.

[7] De Nicola, R., Ferrari, G. and Pugliese, R., Klaim: a Kernel Language for Agents Interaction and Mobility, IEEE Trans. on Software Engineering, Vol.24(5), 1998.

[8] Fournet, C., Gonthier, G., Lévy, J.-J., Maranget, L., and Rémy, D., A Calculus for Mobile Agents, CONCUR'96, LNCS 1119, pp.406-421, Springer-Verlag, 1996.

[9] Gunter, C., Semantics of Programming Languages: Structures and Techniques, MIT Press, 1992.

[10] Java, Sun Microsystems Inc., http://www.javasoft.com/.

[11] Heintze, N. and Riecke, J., The SLam Calculus: Programming with Secrecy and Integrity, POPL'98, pp.365-377. ACM Press, 1998.

[12] Hennessy, M. and Riely, J., Resource Access Control in Systems of Mobile Agents, CS Report 02/98, University of Sussex, http://www.cogs.susx.ac.uk, 1998.

[13] Honda, K., Composing Processes, POPL'96, pp.344-357, ACM, 1996.

[14] Honda, K., A Theory of Types for the pi-calculus, pp.112, Typescript. November, 1998. Available at http://www.dcs.qmw.ac.uk/ kohei.

[15] Honda, K., Vasconcelos, V. and Yoshida, N, Secure Infor- mation Flow as Typed Process Behaviour, ESOP'00, LNCS 1782, pp.180-199, Springer, 2000.

[16] Honda, K. and Yoshida, N., Combinatory Representation of Mobile Processes. POPL'94, pp.348-360, ACM Press, 1994.

[17] Honda, K. and Yoshida, N., On Reduction-Based Process Semantics. TCS, pp.437-486, No.151, North-Holland, 1995.

[18] Kirli, D., A static type system for detecting potentially transmissible functions, ECOOP Workshop MOS'99, 1999.

[19] Kobayashi, N., Pierce, B. and Turner, D., Linearity and the pi-calculus, POPL'96, ACM Press, 1996.

[20] Kobayashi, K., Nakade, M. and Yonezawa, A., Static analysis of communication for asynchronous concurrent programming languages, SAS'95, LNCS 983, Springer, 1995.

[21] Milner, R., Polyadic $\pi$-Calculus, Logic and Algebra of Specification, Springer, 1992.

[22] Milner, R., Parrow, J.G. and Walker, D.J., A Calculus of Mobile Processes. Infor. \& Comp., 100(1), pp.1-77, 1992.

[23] Necula, G., Proof-carrying code. POPL'96, ACM, 1996.

[24] O'Hearn, P., Power, J., Takeyama, M., and Tennent, D., Syntactic control of interference revised, MFPS'97, ENCS, Elsevier, 1997.

[25] ObjectSpace Inc. ObjectSpace Voyager home page, http://www.objectspace.com/voyager.

[26] Pierce, B.C. and Sangiorgi. D, Typing and subtyping for mobile processes. MSCS, 6(5):409-454, 1996.

[27] Pierce, B.C. and Sangiorgi. D, Behavioral Equivalence in the Polymorphic Pi-calculus. POPL'97, ACM Press, 1997.

[28] Riely, J. and Hennessy, M., Trust and partial typing in open systems of mobile agents. POPL'99, ACM Press, 1999.

[29] Sangiorgi, D., Expressing Mobility in Process Algebras: First Order and Higher Order Paradigms. Ph.D. Thesis, University of Edinburgh, CST-99-93, 1993.

[30] Tang, Y.-M., and Jouvelot, P., Effect systems with subtyping, PEPM'95, ACM Press, 1995.

[31] Tofte, M. and Talpin, J.-P., Region-based memory management, Info. \& Comp., 132(2)109-176, 1997.

[32] Vasconcelos, V., Typed concurrent objects. ECOOP'94, LNCS 821, pp.100-117. Springer-Verlag, 1994.

[33] Vasconcelos, V. and Honda, K., Principal Typing Scheme for Polyadic $\pi$-Calculus. CONCUR'93, LNCS 715, pp.524-538, Springer, 1993.

[34] Vasconcelos, V., Lopes, L. and Silva, F., Distribution and Mobility with Lexical Scoping in Process Calculi, 3rd HLCL, ENTCS 16(3), Elsevier, 1998.

[35] Volpano, D. and Smith, G., Secure information flow in a multi-threaded imperative language, pp.355-364, POPL'98, ACM, 1998.

[36] Yoshida, N., Graph Types for Monadic Mobile Processes, FST/TCS'16, LNCS 1180, pp. 371-386, Springer, 1996.

[37] Yoshida, N. and Hennessy, M., Subtyping and Locality in Distributed Higher Order Processes. CONCUR'99, LNCS 1664, pp.557-573, Springer, 1999.

[38] The full version of this paper, CS Technical Report 02/99, University of Sussex, Available at: http://www.mcs.le.ac.uk/rnyoshida, Nov, 1999. 